\title{
Un nouveau mécanisme de régulation de PTEN par la PI3-kinase
}

\author{
I. Ben Sahra \\ (C) Springer-Verlag France 2011
}

La voie de la phosphatidylinositol-3-kinase (PI3K) est dérégulée dans plusieurs situations pathologiques, notamment dans plusieurs cancers, le diabète, l'obésité et l'insulinorésistance.

La PI3-kinase est constituée d'une sous-unité catalytique, $\mathrm{p} 110 \alpha$, et d'une sous-unité régulatrice, $\mathrm{p} 85 \alpha$. La p85 $\alpha$ joue un rôle important dans la stabilité et la localisation de l'activité enzymatique de la sous-unité p110 $\alpha$. Cette enzyme est responsable de la production de phospholipides clés, les phosphatidylinositol-3,4,5-trisphosphates $\left(\mathrm{PI} 3,4,5 \mathrm{P}_{3}\right)$ nécessaires à la transduction du signal lors d'une stimulation par un facteur de croissance, tel que l'insuline. La phosphatase PTEN déphosphoryle les phospholipides, $\mathrm{PI} 3,4,5 \mathrm{P}_{3}$ en $\mathrm{PI} 4,5 \mathrm{P}_{2}$, diminuant ainsi la transduction du signal, et donc la réponse aux facteurs de croissance. L'étude de Chagpar et al. [1] montre que la sous-unité p85 $\alpha$, par son domaine $\mathrm{BH}$, peut interagir directement avec PTEN induisant ainsi une augmentation de son activité phosphatase. La surexpression d'un mutant de la PI3K incapable de se lier à PTEN conduit à une augmentation du niveau basal de la

I. Ben Sahra

Inserm U895, centre méditerranéen de médecine moléculaire, bâtiment universitaire Archimed,

151, route Saint-Antoine-de-Ginestière, BP 23194, F-06204 Nice cedex 03, France

Université de Nice-Sophia-Antipolis, faculté de médecine, F-06000 Nice, France

Inserm U895, C3M, équipe 7, F-06000 Nice, France phosphorylation de la protéine kinase Akt, activée par la voie PI3K. À travers cette régulation, les auteurs suggèrent que la PI3-kinase peut contrôler son propre niveau d'activité. Cela pourrait expliquer pourquoi les souris invalidées pour l'isoforme p85 $\alpha$ gardent une activité PI3K élevée en utilisant l'isoforme p50 $\alpha$ capable de lier la sousunité p110 [2]. Cependant, contrairement à l'isoforme p $85 \alpha$, la protéine p $50 \alpha$ est incapable de se lier à PTEN. Par conséquent, il a été observé une plus forte activation de la voie PI3K chez les souris p85a -/-, corrélant bien avec le rôle contradictoire de la p95 $\alpha$ consistant soit à optimiser l'activation de la p110 $\alpha$, soit à l'inhiber par l'intermédiaire de PTEN. Le fonctionnement de la sous-unité p85 $\alpha$ doit donc être réévalué, car ce n'est plus seulement un régulateur de la production de $\mathrm{PI} 3,4,5 \mathrm{P}_{3}$ par la $\mathrm{p} 110 \alpha-\mathrm{PI} \mathrm{K}$, mais c'est également un régulateur de la déphosphorylation des $\mathrm{PI} 3,4,5 \mathrm{P}_{3}$ par l'intermédiaire de l'activation de la phosphatase PTEN. Cela est d'autant plus important que la PI3-kinase joue un rôle central dans les voies métaboliques de l'insuline, dont le transport de glucose.

\section{Références}

1. Chagpar RB, Links PH, Pastor MC, et al (2010) Direct positive regulation of PTEN by the $\mathrm{p} 85$ subunit of phosphatidylinositol-3kinase. Proc Natl Acad Sci USA 107(12):5471-6

2. Terauchi Y, Tsuji Y, Satoh S, et al (1999) Increased insulin sensitivity and hypoglycaemia in mice lacking the $\mathrm{p} 85$ alpha subunit of phosphoinositide-3-kinase. Nat Genet 21(2):230-5 